\title{
Sui costrutti esistenziali sardi. Effetti di definitezza, deissi, evidenzialità ."
}

\section{Introduzione}

I costrutti esistenziali sardi sono noti in sede teorica per via degli effetti di definitezza da cui sono caratterizzati (l'alternanza delle copule ESSE e HABERE, la seconda priva di accordo) e, in minor misura, per la presenza di proforme deittiche e neutre. La letteratura esistente su questi costrutti tratta di un ristretto numero di varietà logudoresi e nuoresi (Bentley 2004, Jones 1993, La Fauci/Loporcaro 1993, 1997) oppure del sardo in genere (Remberger 2009). Mancano però trattazioni dettagliate sia dei costrutti esistenziali campidanesi sia dell'estensione geolinguistica delle caratteristiche summenzionate. Con il presente lavoro ci proponiamo di cominciare a colmare questa lacuna, riportando i risultati di un'indagine condotta in dodici località: ${ }^{1}$ Bono, Bonorva, Buddusò, Bitti, Orosei, Orgosolo, Fonni, Terralba, Sardara, Sanluri, Villacidro e Monastir. Secondo la classificazione di Virdis (1988), in questi punti geolinguistici si parla il logudorese sud-orientale (Bono), il logudorese centrale (Bonorva e Buddusò), il nuorese settentrionale (Bitti e Orosei), il nuorese orientale e meridionale (Orgosolo e Fonni) e il campidanese centro-occidentale (Terralba, Sardara, Sanluri, Villacidro e Monastir). I nostri risultati ci permettono di individuare un'area sotto certi aspetti omogenea, che accomuna il logudorese centrale e sudorientale al nuorese settentrionale. In quest' area abbiamo riscontrato l'alternanza delle copule éssere e áere con modalità analoghe, seppur non identiche, a quelle già descritte in letteratura. Più specificamente, éssere è in genere selezionato quando il sintagma nominale postcopulare è definito, con interessanti devianze sistematiche sulle quali torneremo in seguito, e áere quando il sintagma nominale postcopulare è indefinito.

Le due copule si alternano in base agli stessi criteri anche nelle parlate dei meno giovani di due località campidanesi, Sardara e Sanluri. A Villacidro, e marginalmente a Sardara, la selezione di $a i$, al posto della copula non marcata essi, può invece assolvere una funzione evidenziale, cioè la distinzione dei fatti presenti e tangibili dai ricordi e le dicerie. Nelle rimanenti località abbiamo rilevato la generalizzazione dell'una o dell'altra copula, sebbene l'alternanza delle due forme sia emersa, molto sporadicamente, nella parlata dei più anziani di quasi tutti i punti sondati. È di rilievo che punti geolinguistici molto affini in base ad altri criteri (per esempio, i punti del cosidetto gruppo Fonni, Wagner 1907, § 61, 28-29, 78-79) siano caratterizzati dalla generalizzazione di copule diverse (éssere a Fonni, áere ad Orgosolo).

\footnotetext{
* Ringrazio lo Arts and Humanities Research Council (http://www.ahrc.ac.uk/Pages/default.aspx) e la School of Languages, Linguistics and Cultures dell'Università di Manchester per aver reso possibile questa ricerca con il finanziamento AH/E506011/1 e il congedo concessomi nell'anno accademico 2008/09. Sono inoltre immensamente grata ai parlanti nativi che hanno risposto con cordialità, entusiasmo, pazienza e generosità alle mie domande, e agli amici e collaboratori sardi (Francesco Casti, Giuseppe Casti, Elisabetta Cocco, Margherita Dore, Manuela Mereu, Lucia Molinu, Elena Pala, Carminu Pintore, Gianfranco Pintore, Ignazio Putzu, Anna Maria Salis) che mi hanno messo in contatto con i soggetti da intervistare e mi hanno spesso accompagnato di persona durante le interviste. È alla gente sarda che dedico questo lavoro, in segno di ammirazione per la loro fedeltà alla propria lingua.

${ }^{1}$ I dati sono stati raccolti sul campo dall'autrice (nel luglio del 2007 e da ottobre 2008 a marzo 2009) con interviste guidate ad un campione di trenta informanti di due fasce di età (da 17 a 35 anni e da 58 a 93 anni). Per ogni località abbiamo intervistato almeno un rappresentante di ciascuna fascia di età, con l'eccezione di Buddusò (dove abbiamo intervistato solo anziani) e Monastir (solo un giovane). Sebbene il nostro campione non sia rappresentativo dal punto di vista sociolinguistico, nel corso dell'analisi faremo presenti le differenze riscontrate fra i dati offerti dai parlanti dell'una e l'altra fascia di età.
} 
Per quanto riguarda le proforme, $b i$ (< IBI), oltre a denotare distanza dal parlante, può essere una proforma neutra a Bono, Bonorva, Buddusò e Bitti, dove la proforma che indica prossimità al parlante, oppure corrispondenza con o inclusione nella locazione dello stesso, è $(n)$ che $(<\operatorname{HINC}(\mathrm{E}))$. Nei rimanenti punti nuoresi (Orosei, Orgosolo e Fonni), bi è l'unica proforma da noi attestata, ad eccezione di una forma composita binche, usata solo da due parlanti colti di orgolese la cui testimonianza è stata lasciata fuori corpus (si veda $\S 3$ ). Dal punto di vista delle proforme, dunque, l'area del nuorese settentrionale si è presentata fratta: il bittese segue il modello conservativo riscontrato anche nel logudoro, Orosei si associa al tipo innovativo riscontrato anche nel nuorese orientale e meridionale.

In area campidanese $(n) c i \quad(<\operatorname{HINC}(\mathrm{E}))$ indica prossimità al centro deittico dell'enunciazione, mentre la deissi di ddoi / ddui (< ILLOC(QUE)), indicando prevalentemente lontananza dal parlante, si definisce negativamente rispetto allo stesso. $D d u$, uno sviluppo di $d d o i$ (Wagner 1960, 610-611, s.v. iddoi), è una proforma neutra delle varietà di Sardara e Sanluri che alternano essi e ai come accennato sopra, mentre, in queste stesse varietà, ddoi marca il costrutto in senso evidenziale, indicando l'assenza di un'esperienza visiva dell'esistente. Anche a Villacidro ddoi può connotare il costrutto in senso evidenziale, in questo caso riportivo, in concomitanza alla scelta di ai già accennata sopra. Infine, $(n)$ ci può essere una proforma neutra in tutte le varietà campidanesi. La generalizzazione di una copula $o$ proforma, laddove è riscontrata, è totale nella produzione dei giovani, mentre può ancora essere parziale nelle varietà della fascia di età più alta.

I nostri risultati offrono spunti di riflessione sulle forme e le funzioni dei costrutti esistenziali, corroborando l'ipotesi che gli effetti di definitezza, seppur codificati nella morfosintassi, dipendano da vincoli di marcatezza semantici e pragmatici sul soggetto (Beaver/Francez/Levinson 2006, Bentley 2009). I dati sardi, inoltre, rivelano interessanti correlazioni fra la deissi dei costrutti esistenziali e la specificità del pivot (logudorese), ${ }^{2}$ nonché la marcatura della fonte della conoscenza del contenuto proposizionale dell'enunciato (campidanese).

Il lavoro si articola come segue. In primo luogo, discutiamo la distribuzione e la funzione delle copule $(\$ 2)$, in secondo luogo, la distribuzione e le funzioni delle proforme $(\S 3)$. Avanziamo poi qualche ipotesi teorica sui dati riportati ( $§ 4)$. Seguono le conclusioni $(\S 5)$.

\section{Le copule}

Contrariamente ad altre lingue romanze, diverse varietà del sardo non hanno una sola copula esistenziale (come, ad esempio, l'esito di HABERE in francese non letterario, alcune varietà del calabrese moderno, etc., e l'esito di ESSE in italiano moderno, rumeno, etc.), ma due, continuando così l'alternanza di ESSE e HABERE attestata nei

\footnotetext{
${ }^{2}$ Seguendo un uso diffusosi in letteratura a partire da Milsark (1974, 1979), adottiamo il termine pivot con riferimento all'argomento introdotto nell'enunciato dal costrutto esistenziale (si veda la nozione di discourse existence proposta da Abbot 1993, 41). Il pivot è di norma un sintagma nominale, o una forma $w h-$, ma in alcune lingue può anche essere codificato da una forma aggettivale (per esempio il calabrese settentrionale c'è pulitu '(c')è pulito' o l'italiano substandard c'è pieno di gente) o da un'intera proposizione (per esempio, l'italiano Che c'è? C' $\grave{e}$ che sono stanca del tuo comportamento). Nelle nostre interviste non siamo riusciti a ottenere esempi sardi di pivot esistenziali di questo tipo. L'accezione del termine pivot che adottiamo in questa sede va distinta da quella che si riferisce all'argomento omesso di costrutti a controllo come Luca $_{i}$ ha promesso di [pivot $_{i}$ ] andarci.
} 
costrutti esistenziali del latino tardo (Zamboni 2000, 106). Le due copule figurano nei primi testi campidanesi (si vedano gli esempi (1a-d)) e logudoresi (si vedano (2a-b)). ${ }^{3}$

(1) a. Et non apat ausancia iudice et nin donna [...] ki pus nos aet esser. 'E non osi giudice o donna.... che ci sarà dopo di noi.'

(Solmi 1905, 14, Carta I , 6)

b. Conporeilli a Petru [...] sa parzoni cantu bi aeat sua et de fradis suus.

'Comprai a Pietro... la parte sua e di suo fratello che c'era [lit. aveva].'

(Solmi 1905, 23, Carta IX, 10)

c. [...] Hominis bonus ki lloi furunt.

'[...] Uomini buoni che vi furono.'

(Solmi 1905, 29, Carta XIII, 5)

d. Et dedilloi donna Muscu de Unali [...] a sanctu Jorgi [...] omnia cantu illoi aeda in sa billa.

'E donna M. Di U. diede a San J. tutto quanto vi è [lit. ha] nel villaggio.'

(Solmi 1905, 30, Carta XIII, 8)

(2) a. Testes ki ui furun, uue conporai.

'Testimoni che ci furono quando comprai.'

(Delogu 1997, 60)

b. E ppumu e fficu cantu ui aueat.

'Tutti i frutti e i fichi che c'erano.'

(Delogu, 1997, 78)

L'attuale estensione geolinguistica del tipo di costrutto esistenziale in cui si alternano gli esiti di ESSE e HABERE non è nota. Le grammatiche del campidanese menzionano una forma invariabile at (la terza persona singolare di ai 'avere') della quale, però, non vengono specificate né le funzioni né la distribuzione diatopica rispetto alla forma non marcata ( $n c$ ')essi (Blasco Ferrer 1986, 144, Lepori 2001, 61). ${ }^{4}$ L'alternanza sistematica di éssere, accordato, e áere, invariato, nei costrutti esistenziali logudoresi e nuoresi è, invece, un fatto ben noto sin dalle trattazioni di Jones (1993) e La Fauci/Loporcaro (1993, 1997), che riguardano, rispettivamente, le varietà di Lula (nuorese settentrionale) e Bonorva (logudorese centrale) (si veda anche Corda 1994, 64, 66). La nostra indagine ha confermato i risultati di questi studi, indicando che l'alternanza di éssere e áere è tutt'ora la norma nei costrutti esistenziali di Bonorva, ma anche Bono e Buddusò, e, in area nuorese, Bitti ed Orosei. La copula éssere si accorda regolarmente per numero e persona col pivot (si veda (3)), mentre la copula áere non si accorda (si veda (4)). ${ }^{5}$

\footnotetext{
${ }^{3}$ Le antiche carte volgari campidanesi citate in questo lavoro non sono quelle reputate filologicamente inaffidabili da Paulis (1997a). Per qualche problema riguardante la correttezza della trascrizione delle carte campidanesi, incluse alcune di quelle da noi prese in considerazione, si vedano Paulis (1997b) e Blasco Ferrer (2003, 50). Per la difficoltà dell'interpretazione delle carte si veda Paulis (1997c).

${ }^{4}$ Blasco Ferrer $(1988,112-113)$ cita due esempi ogliastrini con TENERE, forma che non compare nei nostri dati di diversa provenienza se non, occasionalmente, in costrutti senza proforma e con l'accordo verbale (si veda $(5 \mathrm{c})$ ).

${ }^{5}$ Per brevità nella trascrizione degli esempi omettiamo talora il contesto in cui questi sono stati prodotti. Ad esempio, i dati in (3a-e) sono stati prodotti nel contesto 'Cosa possiamo usare per fermare quella porta?' , quelli in (4a-e) nel contesto 'Che cosa c'è in quella strada?'. L'accordo per persona è testimoniato, ad esempio, dai dati riportati in (66a-b), in cui ESSE non figura alla terza persona, ma alla seconda e prima persona singolare.
} 
(3) a. Bi sun sos líberos. ${ }^{6}$

b. Bi sun cussos libros.

(Bonorva)

c. Bi son cussos libros.

(Bono)

d. Bi son cussos libros.

(Buddusò)

e. Bi son cussos libros.

'Ci sono i / quei libri.'

(Bitti)

(Orosei)

(4) a. B'at duos bars e una cheža.

(Bonorva)

b. B'at duos bars e una creža.

(Bono)

c. B'at duos tzilleris e una creža.

(Buddusò)

d. B'at duos tzilleris e una creia.

(Bitti)

e. B'at unu tzilleri e una cresia.

(Orosei)

'Ci sono due bar / c'è un bar e una chiesa.'

Occasionalmente áere è accordato con un soggetto non espresso, alternandosi con éssere se il pivot è definito. Si vedano i dati in (5a-b), forniti dallo stesso informatore. I costrutti con HABERE accordato trovano un corrispondente semanticamente e sintatticamente equivalente in quelli con TENERE che figurano altrettanto sporadicamente nel nostro corpus (si veda $(5 \mathrm{c})$ ).

(5) a. Bi son / b'an solu sas medias.

(Bitti)

'Ci sono / hanno le medie.'

b. Sas iscolas chi bi son / b'amus in Bithi.

(Bitti)

'Le scuole che ci sono /abbiamo a Bitti.'

c. Non tenimus mancu išolas.

'Non abbiamo neanche scuole.'

(Fonni)

I costrutti con HABERE e TENERE accordati non smentiscono la generalizzazione di cui sopra sul comportamento delle due copule, visto che si tratta di costrutti biargomentali, diversi da quelli monoargomentali che ci interessano in questo studio. Se, infatti, éssere si accorda con il pivot, áere si accorda con il soggetto non espresso, in (5a-b), com'è dimostrato dalla prima persona plurale in $(5 \mathrm{~b})$.

Per quanto riguarda la funzione dell'alternanza di éssere, accordato, e áere, invariabile, se, in genere, la prima copula è selezionata quando il pivot è definito (si veda (3)), e la seconda quando esso è indefinito (si veda (4), con lettura debole, nel senso di Milsark 1979, dei quantificatori 'due' e 'uno'), abbiamo riscontrato qualche devianza sistematica da questa tendenza. Più specificamente, mentre éssere è stato selezionato con una classe di pivot formalmente indefiniti ('solo un(a) x'), áere non è mai comparso in contesti definiti, se non nei rari esempi del tipo di costrutto

\footnotetext{
${ }^{6}$ Adottiamo i principi di trascrizione ortografica esposti in Pittau (1978), con le seguenti modifiche e aggiunte: (i) usiamo il digramma $c h$ per / $/$ / davanti a vocale palatale; (ii) non usiamo il grafema $j$; (iii) non indichiamo in nessun caso la vocale paragogica né gli scempiamenti nei risultanti contesti intervocalici; (iv) con $\check{z}$ indichiamo una fricativa (pre)palatale sonora, con $\check{s}$ la fricativa prepalatale sorda che è esito di /sk/ a Fonni (si vedano Contini 1987, 124-125, Wagner 1941, § 281, 174, che però sostiene che essa ricorra soltanto davanti a /u/, e Wolf 1985, 279 che solleva il problema dell'identificazione dei contesti di ricorrenza di questo esito di $/ \mathrm{k} /$ ). Il lettore noti che non modifichiamo l'ortografia degli esempi riportati dai testi e non correggiamo le tracce della pressione dell'italiano che sono emerse nella raccolta dei dati (per esempio, la forma studenti 'studenti' riportata in (15a-b) e (17b) al posto di istudiantis).
} 
esemplificato sopra in (5a-b). ${ }^{7}$ Inoltre, la scelta di éssere ricorre nelle interrogative con 'quale' e 'chi', diversamente da quelle con 'quanto' e 'che cosa'. Questi risultati sono illustrati negli esempi da (6) a (10). Le due alternative date in uno stesso esempio indicano che copule diverse sono state scelte nello stesso contesto da informatori diversi o, più raramente, dallo stesso informatore. ${ }^{8}$ Indichiamo invece con éssere o áere il caso in cui una sola copula è stata scelta nel contesto dato. ${ }^{9}$

(6) a. B'at / B'est solu un'ómine in sa vida mea.

(Bonorva)

'C'è solo un uomo nella mia vita.'

b. B'at / b'est un'isveglia chi funtzionat.

(Bono)

'C'è una sola sveglia che funziona bene.'

c. B' at / b'est petzi un'istudente in sa letzione.

(Bitti)

d. B'est solu un dischente in iscola.

(Orosei)

'C'è solo uno studente a lezione.'

(7) a. Cales istudentes bi sun / b'at?

(Bonorva)

b. Cales alunnos bi sun / b'at?

(Bono)

c. Cales bi son?

(Buddusò)

d. Cales istudentes b'in / b'aiat in sa letzione?

(Bitti)

e. Cales dischentes b'in / b'aiat in sa letzione?

(Orosei)

'Quali (studenti) ci sono / c'erano (a lezione)?'

(8) a. Chie b'est? / b'at?

(Bonorva)

b. Chie b'est? / b'at?

(Bono)

c. Chie b'est in cussa carrera?

(Buddusò)

d. Chie b'at?

(Bitti)

e. Chie b'est in domu?

(Orosei)

'Chi c'è (a casa / in quella strada)?'

(9) a. Cantos istudentes b'at?

(Bonorva)

b. Cantos alunnos b'at?

(Bono)

c. Cantos istudentes b'at?

(Buddusò)

d. Cantos istudentes b'aiat in sa letzione?

(Bitti)

e. Cantos dischentes bi son / b' aiat in sa letzione?

(Orosei)

'Quanti studenti ci sono /c'erano (a lezione)?'

(10) a. Itte b'at in cussu istradone?

(Bonorva)

b. Itte b'at in cussu istradone?

(Bono)

c. Itte b'at in cussu istradone?

(Buddusò)

d. Itte b'at in cussu caminu?

(Bitti)

e. Itte b'at in cudda carrera?

(Orosei)

\footnotetext{
${ }^{7}$ Avendo trovato sul web qualche esempio di costrutti monoargomentali con áere invariato e un pivot formalmente definito, abbiamo sottoposto questi dati al giudizio dei nostri informatori, i quali hanno immancabilmente suggerito di sostituire áere con éssere accordato.

${ }^{8}$ Per contesto non si intende soltanto il costrutto dato nell'esempio, ma l'insieme dei costrutti in cui figura ciascuna classe di pivot pertinente (solo un(a) $\mathrm{x}$, quale, chi, quanto, che cosa).

9 I dati raccolti a Buddusò non sono probanti per il contesto illustrato in (6), perché le informatrici hanno modificato il pivot nei contesti pertinenti (per esempio, la forma b'est maridu meu, lett. c'è mio marito, è stata fornita nel contesto b'at / b'est solu un'ómine in sa vida mea, 'c'è solo un uomo nella mia vita'). Si veda, però, il seguente esempio, formulato dalle stesse informatrici, dove figura éssere: unu mattulu 'e fiores chi prima non (n)che fit 'un mazzo di fiori che prima non c'era'.
} 
'Che cosa c'è in quella strada?'

La scelta della copula sembra anche essere marginalmente affetta alla deissi spaziale del costrutto, ma su questo punto ci soffermeremo in seguito $(\S 4)$.

Oltre alle devianze sistematiche di cui sopra, i dati raccolti ad Orosei presentano qualche riccorrenza di éssere che non si presta a paralleli con i risultati delle altre varietà nuoresi settentrionali e delle varietà logudoresi, ma farebbe pensare ad una tendenza alla generalizzazione di éssere.

(11) a. B'in a bottu bestias.

(Orosei)

'C'erano tanti animali.'

b. B'it frittu e iscuru.

'C'era freddo e buio.'

c. B'est su prus de sos cumpanzos tuos.

'C'è la maggior parte di tuoi amici.'

L'alternanza sistematica di ESSE, accordato, e HABERE, invariato, si riscontra anche nelle varietà dei meno giovani di due località campidanesi, Sardara e Sanluri. Anche in questo caso la scelta della copula sembra dipendere dalla definitezza del pivot.

(12) a. La' ca ddu at omi bussendi sa porta.

$(\text { Sardara }-\mathrm{H})^{10}$

b. Ddu at omi in sa porta.

(Sanluri - H)

'C'è un uomo (che bussa) alla porta.'

(13) a. Ddu est su postinu.

(Sardara - H)

b. Ddui est / (N)c'est su postinu.

(Sanluri - H)

'C'è il postino.'

Essi figura anche sistematicamente nei contesti illustrati in (6), (7) e (8) (cf. (14), (15) e (16)), ma non nel contesto illustrasto in (9) (cf. (17)) e solo a Sanluri in quello esemplificato in (10) (cf. (18)).

(14) a. (N)c'est ua femia sceti in sa vida mia.

(Sardara - H)

b. $(\mathrm{N}) \mathrm{c}$ 'est feti ua femia in sa vida mia.

(Sanluri - H)

'C'è una sola donna nella mia vita.'

(15) a. Cali funt is studenti chi (n)ci funt?

(Sardara - H)

b. Cali studenti ddui funt?

(Sanluri - H)

'Quali studenti ci sono?'

(16) a. Chi (n)c'est?

(Sardara - H)

b. Chi ddui est?

(Sanluri - H)

'Chi c'è?'

(17) a. Cantu studentis (n)c'at?

(Sardara - H)

b. Cantu studenti ddui at?

(Sanluri - H)

'Quanti studenti ci sono?'

${ }^{10}$ Con H (high) ci riferiamo ai parlanti della fascia di età alta, con L (low) ai parlanti della fascia di età più bassa. 
(18) a. Ita (n)c'at?

(Sardara - H)

b. Ita ddui est?

(Sanluri - H)

'Che cosa c'è?'

Infine, essi coricorre con il pivot 'nessuno', ma non con 'niente'.

(19) a. No ddu est / (n)c'est nemus.

(Sardara - H)

b. No ddu est nisciunus.

(Sanluri - H)

'Non c'è nessuno.'

(20) a. No ddu at / (n)c'at nudda.

(Sardara - H)

b. No ddu at nudda.

(Sanluri - H)

'Non c'è niente.'

Essi è la copula non marcata nei dati prodotti dai giovani delle stesse località campidanesi. Si veda l'esempio (21), che contrasta con il corrispettivo fornito dai parlanti non giovani (cf. (12)).

(21) a. (N)c'est u omi in sa porta.

(Sardara - L)

b. $(\mathrm{N}) c$ 'est $\mathrm{u}$ omi in sa porta.

(Sanluri - L)

'C'è un uomo alla porta.'

Va tuttavia precisato che, a Sanluri, essi è stato selezionato dai giovani in tutti i contesti ad eccezione dei due riportati in (22a-b), che contrastano con (23a-b).

(22) a. Ita (n)c'at?

(Sanluri - L)

'Che cosa c'è?'

a. No (n)c'at nudda.

'Non c'è niente.'

(23) a. Chi (n)c'est?

(Sanluri - L)

'Chi c'è?'

b. No (n)c'est nemus.

'Non c'è nessuno.'

Invece, a Sardara ai coricorre sporadicamente con la proforma ddoi marcando, secondo il parere spontaneamente offerto dall'informatrice giovane, l'assenza di un'esperienza visiva dell'esistente.

(24) a. Ddoi at su propriu problema in Sicilia.

(Sardara - L)

'C'è lo stesso problema in Sicilia.'

b. Chi ddoi at?

'Chi c'è?' [contesto: non si vede nessuno]

(25) a. No (n)c'est nemus.

(Sardara - L)

'Non c'è nessuno.' [qui]

b. No ddoi at nemus.

'Non c'è nessuno.' [altrove] 
(26) a. No (n)c'est nudda.

(Sardara - L)

'Non c'è niente.' [qui]

b. No ddoi at nudda.

'Non c'è niente.' [altrove]

L'alternanza delle copule in base al modo in cui il locutore è venuto a conoscenza del contenuto proposizionale dell'enunciato è stata riscontrata anche nei dati forniti dai meno giovani di Villacidro. Più specificamente, la selezione di $a i$, in concomitanza con $d d o i$, al posto della copula non marcata essi, può esprimere una funzione evidenziale prevalentemente riportiva, cioè la distinzione dei fatti personali o tangibili dalle dicerie e i ricordi.

(27) a Ddoi est su stessu problema in Sicilia.

(Villacidro- H)

'C'è lo stesso problema in Sicilia.'

b Ddoi at su stessu problema in Sicilia.

'[Si dice che] ci sia / ci fosse lo stesso problema in Sicilia.'

(28) a. No (n)c'est nemus.

'Non c'è nessuno.' [verificato di persona]

(Villacidro- H)

b. No ddoi at nemus.

'Non c'è nessuno.' [racconto]

(29) a. No (n)c'est nudda.

'Non c'è niente.' [verificato di persona]

(Villacidro- H)

b. No ddoi at nudda.

'Non c'è niente.' [racconto]

(30) a. (N)ci funt medas problemas.

(Villacidro- H)

'Ci sono / abbiamo molti problemi.'

b. Ddoi at medas problemas.

'Ci sono molti problemi.' [racconto, diceria su problemi di altri]

Ritorneremo in seguito sulla correlazione fra la copula e la proforma, riscontrata sia nei dati offerti dall'informatrice giovane di Sardara, sia in quelli dei meno giovani di Villacidro. In questa sede è il caso di ribadire che essi è la copula non marcata nelle varietà Sardara $(\mathrm{L})$ e Villacidro $(\mathrm{H})$. Infatti, questa copula ricorre a prescindere dalla definitezza del pivot, figurando anche in contesti che potrebbero prestarsi ad una lettura marcata nel senso evidenziale di cui sopra. Si vedano i risultati in (31), forniti dall'informatrice più anziana di Villacidro.

(31) a. No appu biu is froris chi ddoi funt in cui.

(Villacidro- H) 'Non ho visto i fiori che ci sono lì.'

b. Ddoi est u omi in sa porta.

'C'è un uomo alla porta.'

c. No ddoi funt mancu scolas.

'Non ci sono neanche scuole.'

d. (N)c'est su posteri.

'C'è il postino.' 
Sia nella produzione dei giovani di Villacidro sia nelle rimanenti varietà campidanesi e nuoresi, abbiamo rilevato la generalizzazione dell'una o dell'altra copula, sebbene l'alternanza delle due forme sia emersa, molto sporadicamente, nella parlata dei più anziani di quasi tutti i punti sondati (si veda, ad esempio, (35a)). ESSE si è generalizzato a Terralba, Monastir, ${ }^{11} \mathrm{e}$, in area nuorese, Fonni, HABERE ad Orgosolo.

(32) a. Ita ddoi est in cussa strada?

b. Ita ddoi est in cussa strada?

c. Ita ddoi / (n)c'est in cussa strada?

d. Itte b'est in quthu qaminu?

e. Itte b'at in qussu qaminu? 'Che cosa c'è in quella strada?'

(Villacidro- L)

(Terralba)

(Monastir)

(Fonni)

(Orgosolo)

(33) a. (N)ci funt ua pariga 'e domus e ua cresia.

(Villacidro- L)

b. (N)ci / Ddoi funt ua domu e ua cresia.

(Terralba)

c. Ddoi funt unas cantu domus e ua cresia.

(Monastir)

d. Bi sunis duos tzilleris e una cresia.

(Fonni)

e. B'at duos tzilleris e una lqesia.

(Orgosolo)

'Ci sono alcune case / due bar e una chiesa.'

(34) a. (N)ci /ddoi est su postinu.

(Villacidro- L)

b. (N)ci /ddoi est su posteri.

(Terralba)

c. Ddoi est su postinu.

(Monastir)

d. B'est su postinu.

(Fonni)

e. B'at su postinu.

(Orgosolo) 'C'è il postino.'

L'informatore non giovane di Fonni ha selezionato ESSE in tutti i contesti dati con l'unica eccezione di quelli con il clitico $n d(e)$ (> INDE, cf. l'italiano ne) (cf. (35a)). Inotre, non ha accordato ESSE in alcuni dei contesti con pivot indefinito (cf. (35b-c)).

(35) a. A mind'at frores in su pradu?

(Fonni - H)

'Ce ne sono fiori nel prato?'

b. Qistiones bi est?

'Ci sono problemi?'

c. Bardia qantu ovos b'est in frigu.

'Guarda quante uova ci sono in frigo.'

(Fonni - H)

(Fonni - H)

Torneremo alle copule esistenziali nella sezione 4, con qualche riflessione sulla distribuzione funzionale illustrata sopra.

\section{Le proforme}

Anche per quanto riguarda le proforme, parecchie varietà del sardo si distinguono da altre lingue romanze (si veda il francese $y$, il catalano $h i$, etc.), alternando due varianti,

\footnotetext{
${ }^{11}$ Con riferimento alla varietà di Monastir, il lettore ricordi che i nostri risultati sono stati prodotti da un solo informatore giovane.
} 
una delle quali è marcata in senso deittico. ${ }^{12}$ La distribuzione diatopica e funzionale di queste varianti non divide l'area settentrionale nello stesso modo in cui la divide quella delle copule. Infatti, contrariamente a quanto riscontrato nell'analisi delle copule $(\S 2)$, il nuorese settentrionale è disomogeneo: il bittese si associa al modello conservativo del logudoro, mentre Orosei presenta il tipo innovativo riscontrato anche nel nuorese orientale e meridionale. Nel campidanese, invece, abbiamo individuato lo stesso nucleo di conservatività a Sardara e Sanluri. Tuttavia, insieme alla varietà $\mathrm{H}$ di Villacidro, quelle di Sardara e Sanluri offrono anche prove di una tendenza alla marcatura evidenziale non etimologica dei costrutti esistenziali. ${ }^{13}$

Le proforme attestate a Bono, Bonorva, Buddusò e Bitti sono bi $(<\mathrm{IBI}$, Wagner 1960, 610, s.v. ibi) e (n)che (<HINC(E), Wagner 1960, 624, s.v. inke). Al pari di qui/qua in italiano (Vanelli 1972, 18), (n)che è una forma deittica collegata positivamente, in tre modi diversi, col centro deittico costituito dal parlante: coincidenza, inclusione, prossimità al momento dell'enunciazione.
a. In custu istradone (n)ch'at duos bars [...] e una creža.
(Bono)
b. In custu istradone (n)ch'at duos bars [...] e una cheža.
(Bonorva)
c. In custa carrera (n)ch'at duos tzilleris [...] e una creža.
(Buddusò)
'In questa strada ci sono due bar e una chiesa.'
d. Inoche non ( $n$ )ch' at nudda.
(Bitti - H)
'Qui non c'è niente.'

Gli enunciati in (36a-d) ricorrerebbero appropriatamente nel caso in cui il parlante si trovasse nella strada (cf. (36a-c)) o nel luogo (cf. (36d)) di cui parla, ma anche nel caso in cui si riferisse ad una strada o ad un luogo a sé prossimo al momento dell'enunciazione.

La proforma $b i(<\mathrm{IBI})$, invece, può individuare un luogo definito negativamente rispetto al centro deittico costituito dal parlante (cf. (37a-d)) (si veda Vanelli 1972, 18 su lìlà in italiano) oppure ricorre come forma deitticamente neutra. Questa seconda accezione sembrerebbe l'unica ammessa dall'informatore giovane di Bitti, che, diversamente da quello non giovane, non ha dimostrato alcuna competenza (attiva) della proforma $(n)$ che (cf. (38a-b)).
a. In cussu istradone b'at duos bars [...] e una creža.
(Bono)
b. In cussu istradone b'at duos bars [...] e una cheža.
(Bonorva)
c. In cussa carrera b' at duos tzilleris [...] e una creža.
(Buddusò)
'In quella strada ci sono due bar e una chiesa.'
d. In cue non b'at nudda.
(Bitti - H)
'Lì non c'è niente.'

\footnotetext{
${ }^{12}$ Anche l'italiano ha due proforme esistenziali ( $c i<$ ECCE HIC, $v i<\mathrm{IBI}$ ), ma il contrasto semantico fra $c i$ 'qui' e $v i$ 'lì' è andato da tempo perduto (Migliorini 1960, 540). Per il complesso problema dell'identificazione del termine marcato nelle coppie deittiche si veda Ricca $(1993,27-29)$. Nel caso delle coppie di proforme esistenziali discusse in questo contesto, le inferenze deittiche ricavabili dal termine che definiamo non marcato (bi per il logudorese e il nuorese e $(n)$ ci per il campidanese) sono distruttibili. Quelle ricavate dall'uso del termine marcato $(n)$ che (logudorese e la sottovarietà $\mathrm{H}$ del bittese) non sono distruttibili, mentre quelle ricavate dall'uso di ddoi / ddui (campidanese di Sardara e Villacidro) possono essere sostituite da inferenze di tipo evidenziale pienamente concordanti con la deissi di ddoi / ddui.

${ }^{13}$ Blasco Ferrer cita le proforme esitenziali ancá (1986, 144, 214, nota 101) e i(é) (1988, 112), quest'ultima del baunese, di cui non abbiamo riscontrato traccia nelle varietà da noi indagate. Per una lista esauriente delle varianti delle proforme esistenziali sarde si veda Remberger $(2009,231)$.
} 
(38) a. In cussu erettu non b'at mancu iscolas.

(Bitti - L)

b. In custu erettu non b'at mancu iscolas.

(Bitti - L)

'In quel / questo luogo non ci sono neanche scuole.'

Forme derivate da HINC(E) e IBI ricorrono anche nei testi logudoresi antichi, sebbene soltanto la seconda figuri in contesti inequivocabilmente esistenziali. Con riferimento agli esiti di IBI si vedano gli esempi (2a-b), dove la grafia $u i$ indica la forma atona di IBI (Wagner 1960, 610, s.v. ibi). L'altra forma è illustrata qui di seguito.

(39) a. E iusseruninkela a cCoraso.

'E l'avevano portata [lontano da me] a Coraso.'

(Delogu 1997, 90)

b. Bennerun e ffuraruninkela kene lis la dare nen donnu...

'Vennero e me la rubarono [la rubarono da me] senza che gliel'avesse data né il padrone...'

(Delogu 1997, 90)

La discrepanza fra le testimonianze dei due informatori di Bitti indurrebbe a pensare che l'alternanza fra $b i$ e $(n)$ che sia un tratto conservativo e che $b i$ sia in via di generalizzazione. $(N)$ che è d'altro canto del tutto assente negli esempi di costrutti esistenziali forniti da tutti gli informatori di Orosei (cf. (40)) e delle altre varietà nuoresi, Orgosolo (cf. (41)) e Fonni (cf. (42)).

(40) a. In cussu caminu b'at unu tzilleri e una cresia.

(Orosei)

b. In custu caminu b'at unu tzilleri e una cresia.

(Orosei)

In quella / questa strada c'è un bar e una chiesa.'

(41) a. In qussu qaminu b'at duos tzilleris [...] e una lqesia.

(Orgosolo)

b. In qustu qaminu b'at duos tzilleris [...] e una lqesia.

(Orgosolo)

'In quella / questa strada ci sono due bar e una chiesa.'

(42) a. In quthu qaminu bi sunis duos tzilleris e una cresia.

(Fonni)

b. In qustu qaminu bi sunis duos tzilleris e una cresia.

(Fonni)

'In quella / questa strada ci sono due bar e una chiesa.'

È infine degna di nota una forma composita binche riscontrata nella varietà di due parlanti colti di orgolese, residenti a Orosei, che non abbiamo incluso nel campione discusso alla nota $1 .{ }^{14}$ La proforma in questione si alterna con $b i$, marcando una locazione diversa da quella del parlante, contrariamente a $(n)$ che nelle varietà di Bono, Bonorva, Buddusò e Bitti $(\mathrm{H})$.

(43) a. Non b'at nemos [contesto: qui].

(nuorese-fuori corpus)

b. Non binch'at nemos [contesto: altrove].

(nuorese-fuori corpus)

'Non c'è nessuno qui / altrove.

\footnotetext{
${ }^{14}$ I due informatori, di 69 e 24 anni, sono padre e figlio. La loro varietà rivela anche una netta tendenza alla generalizzazione di HABERE, da cui traspare la pressione del modello di Orgosolo su quello di Orosei.
} 
(44) a. Sas iscolas chi b'at inoche.

(nuorese-fuori corpus)

b. Sas iscolas chi binch'at in cue.

'Le scuole che ci sono qui / lì.'

(nuorese-fuori corpus)

(45) a. A bi so ca in cue binch'at nive. 'A quanto pare lì c'è la neve.'

b. Inoche b'at nive: l'apo bida deo.

'Qui c'è la neve: l'ho vista io.'

(nuorese-fuori corpus)

(nuorese-fuori corpus)

Nella stessa varietà è $b i$ la forma scelta in contesti deitticamente neutri.

(46) a. Non bi sunt cuddos libros in sa mesa.

(nuorese-fuori corpus)

'Non ci sono quei libri sul tavolo.'

b. B'at sa prus parte manna de sos amicos tuos. (nuorese-fuori corpus)

'C'è la maggior parte dei tuoi amici.'

Sebbene $b i$ sia il termine deitticamente non marcato, come nelle altre varietà nuoresi e logudoresi a noi note, l'alternanza illustrata in (43)-(45) è singolare per via della deissi di binche, che sembrerebbe contrastare sia con l'etimologia di $(n)$ che sia con i risultati illustrati in (36a-d).

La deissi di $(n)$ che si rivela tuttavia molto più complessa di quanto non si penserebbe in base a dati come quelli in (36a-d) se la si esamina in contesti non esclusivamente esistenziali. A questo proposito si osservino i dati qui di seguito. ${ }^{15}$

(47) a. S'idea fiat de nos nche ghirare oe etotu.

'L'idea era quella di tornarcene oggi stesso.'

b. Apo abertu sa gianna e mi nche so essida iscudèndela.

'Ho aperto la porta e me ne sono uscita sbattendola.' [racconto]

c. In uve nche seis?

'Dove siete?' [al telefono]

d. V. cheret isòrvere s'istamentu [...] e de nche catzare a Istene e a tie.

'V. vuole sciogliere il parlamento e espellerne te e Istene.'

e. E est capassu puru chi nche lu cravent a galera.

'E può darsi persino che lo inchiodino in galera.'

(Pintore 2007, 40, 20, 9, 43)

I costrutti in (47a-e) suggeriscono che la semantica di $(n)$ che sia paragonabile a quella del verbo deittico di movimento come 'venire' nell'opposizione itivo/ventivo dell'inglese (si vedano Fillmore 1975, Huang 2007, 161, Ricca 1993, 39-43): (n)che può infatti denotare la locazione del parlante, o quella dell'interlocutore, al momento dell'enunciazione (cf. $(47 \mathrm{a}, \mathrm{c})$ ), o in un momento diverso, nel qual caso si può trattare di una home base (cf. $(47 \mathrm{~b}, \mathrm{~d}),{ }^{16}$ ma può anche denotare una locazione diversa da quella dei locutori (cf. (47e)). In questo caso, (n)che indica la locazione di un argomento topicale o un personaggio saliente nella narrazione. La semantica di binche nei costrutti esistenziali della varietà esemplificata in (43)-(45), oltre ad essere

\footnotetext{
${ }^{15}$ Per la tendenza del sardo ad accompagnare il verbo con clitici che anticipano o sostituiscono la realizzazione lessicale di tutti i suoi argomenti (quindi non solo quelli con funzione sintattica di soggetto e oggetto) si veda Virdis (2003).

16 La home base (si veda, ad esempio, Ricca 1993, 41) è una locazione con cui un locutore è convenzionalmente associato (la casa, il posto di lavoro, etc.).
} 
spiegabile alla luce della deissi di $b i$, non è dunque del tutto contrastante con la deissi di $(n)$ che.

Per quanto riguarda il campidanese, in tutte le varietà da noi prese in esame si alternano due proforme esistenziali già attestate nei primi testi campidanesi: ddoi / $d d u i$ < ILLOC(QUE) (Wagner 1960, 610-611, s.v. iddói) e $(n) c i<\operatorname{HINC(E)~(Wagner~}$ 1960, 624, s.v. ínke). Solo la prima (illoi) figura in contesti esistenziali già in fase antica (cf. (1c-d)), mentre la seconda, più rara, accompagna predicati di movimento, analogamente a quanto succede in logudorese antico (cf. (39a-b)).

(48) Et falatsi 'nki fini a flumini.

'E scende [da qui] fino al fiume.'

(Solmi 1905, 34, Carta XIV, 8)

Si osservi inoltre che il campidanese antico conosce la proforma esistenziale IBI (cf. (1b)), ma questa sembra essere andata perduta nelle varietà moderne da noi studiate (sulla perdita di questa forma nella storia del campidanese, si veda anche Wagner 1960, 610, s.v. ibi).

Nelle nostre varietà, la proforma $d d o i / d d u i$ è marcata deitticamente, indicando una locazione diversa dalla home base, oppure dalla locazione contingente, del parlante, mentre $(n)$ ci può indicare coincidenza, inclusione o prossimità al centro deittico del parlante, ma svolge anche il ruolo di proforma deitticamente neutra. Per brevità riportiamo solo dati rilevati a Terralba, la località più a nord fra quelle visitate, e Monastir, quella più a sud.

(49) a. Calincua scola ca (n)c'est a Terralba.

(Terralba - H)

b. Calincua scola ca ddoi est a Villacidro.

(Terralba - H)

'Qualche scuola che c'è a Terralba / Villacidro.'

(50) a. Ua pariga 'e scolas ca (n)ci funt innoi.

(Monastir)

b. Ua pariga 'e scolas ca ddoi funt ingunis.

(Monastir)

'Qualche scuola che c'è qui / lì.'

$(N) c i$ è nettamente prevalente nelle varietà dei giovani intervistati a Terralba, Sanluri e Villacidro, ricorrendo anche in contesti contrastanti con la deissi di HINC(E) (cf. (51a-c)), mentre la scelta di $(n) c i$ e ddoi è in genere canonica nei dati forniti dai giovani di Sardara e Monastir (si vedano, ad esempio, (50a-b)).

(51) a. In sa domu de nonnu miu (n)ci fiat ua ziminera.

(Sanluri - L)

b. In sa domu de nonnu miu (n)ci fiat ua ziminera.

(Villacidro - L)

'In casa di mio nonno c'era un camino.'

c. In cui/innoi (n)c'est genti meda.

(Terralba - L)

'Lì / qui c'è molta gente.'

Alla luce di dati come quelli in (51) non si può escludere che sia in atto una tendenza alla generalizzazione di $(n) c i$, il termine non marcato nelle varietà campidanesi con l'alternanza deittica di $(n) c i$ 'qui' e ddoi 'lì'. La generalizzazione di nci potrebbe comportare la perdita dell'alternanza in questione.

Si osservi tuttavia che la disponibilità di una proforma deitticamente neutra non implica di per sé la perdita dell'alternanza delle proforme deittiche. In campidanese esiste infatti un'altra proforma deitticamente neutra, $d d u$, sviluppo di $d d o i$ / ddui 
(Wagner 1960, 610-611, s.v. iddói). Sebbene questa sia citata dalle grammatiche del campidanese moderno (Lepori 2001, 61), l'abbiamo rilevata soltanto nelle varietà più conservative, cioè quelle di Sardara e Sanluri con l'alternanza sistematica delle copule (§ 2).

(52) a. Ddu est su postinu.

(Sardara - H)

'C'è il postino.'

b. Ddu at u omi chi no conosciu.

(Sardara - H)

'C'è un uomo che non conosco.'

(53) a. No ddu est nisciunus.

(Sanluri - H)

'Non c'è nessuno.'

b. No ddu at nudda.

(Sanluri - H)

'Non c'è niente.'

I costrutti in (52) sono stati entrambi prodotti nel contesto 'qualcuno sta bussando alla porta', quelli in (53) entrambi fuori contesto. In contesti deitticamente marcati gli stessi informatori che hanno fornito questi dati hanno scelto, secondo i casi, $(n) c i$ o ddoi / ddui.

(54) a. Ddoi fuit sa giminera in domu 'e nonnu.

(Sardara - H)

'C'era il camino a casa di mio nonno.'

b. No ti preoccùpisti: (n)ci seu deu.

(Sardara - H)

'Non ti preoccupare: ci sono io.'

(55) a. In domu 'e nonnu ddui fuit ua giminera. ${ }^{17}$

(Sanluri - H)

'C'era il camino a casa di mio nonno.'

b. No ti preoccùpisti: (n)ci seu eu.

'Non ti preoccupare: ci sono io.'

(Sanluri - H)

I parlanti giovani di Sardara e Sanluri, al pari degli informatori delle altre località, non hanno usato né riconosciuto la proforma $d d u .{ }^{18}$ Presumiamo dunque che $d d u$ sia il risultato di un precedente processo di erosione formale e funzionale di $d d o i$ / ddui, che non ha tuttavia eliminato dal sistema né la forma di partenza né il contrasto deittico con $(n) c i$. La forma neutra $d d u$ è poi caduta in disuso in tutti i punti sondati con l'eccezione del nucleo di conservatività di Sardara e Sanluri.

Nelle varietà conservative di Sardara e Sanluri ddoi / ddui può anche marcare il costrutto in senso evidenziale, indicando, secondo commenti spontaneamente offerti dai nostri informatori, la non testimonialità oppure l'assenza di un'esperienza visiva concomitante con l'enunciazione. Si vedano gli esempi (24a-b), (25b), (26b), (54a) e (55a), oltre a quelli riportati qui di seguito.

(56) a. A cantu parit ddoi depit ai ni in cui.

(Sardara - H) 'A quanto pare lì c'è la neve.'

b. Castia ca in frigurìferu ddoi depit ai ottu ous. 'Vedi che in frigo ci devono essere otto uova.'

(Sardara - H)

\footnotetext{
${ }^{17} \mathrm{Si}$ veda la discussione dei dati in (6) e (14) per la scelta della copula ESSE in questo costrutto con pivot indefinito.

${ }^{18}$ Tranne il giovane di Terralba che ha dichiarato di riconoscerla ma non usarla. L'informatore anziano di Sanluri, fornendo i dati in (53a-b), ha precisato che $d d u$ era la forma usata dal nonno.
} 
(57) a. Inguddei parit ca ddui est sa ni.

(Sanluri - H)

'A quanto pare lì c'è la neve.'

b. Ddui est su propriu problema in Sicilia.

'C'è lo stesso problema in Sicilia.'

(Sanluri - H)

Nella sezione 2 si notava che, a Sardara, la selezione della copula ai può assolvere la stessa funzione evidenziale che qui si attribuisce alla scelta si ddoi. Nel complesso, i dati raccolti suggeriscono che la marcatura evidenziale del costrutto esistenziale compete principalmente alla proforma, visto che sia i parlanti giovani che quelli non giovani selezionano ddoi in contesti marcati in senso evidenziale, mentre la selezione della copula marca, nella varietà degli anziani, i parametri semantici e pragmatici associati alla definitezza del pivot (si veda anche § 4). Lo stesso può dirsi della marcatura evidenziale dei costrutti esistenziali di Villacidro. I dati offerti dai non giovani di questo paese suggeriscono che solo alcuni marcano l'evidenzialità con la copula $a i$, mentre tutti i non giovani selezionano ddoi per la stessa funzione, cioè la distinzione dei ricordi e le dicerie dall'esperienza presente e di prima mano. Si vedano (27b), (28b), (29b) e (30b), oltre agli esempi in (58).

a. In domu de aiàia ddoi fiat sa ziminera. 'C'era il camino a casa di mio nonno.'

b. Castia cantu ous ddoi est in frigu.

(Villacidro - H)

'Guarda quante uova ci sono in frigo.'

c. Ddoi abiat scuru e frius.

'C'era freddo e buio.' [contesto: un sogno]

Dello stesso tipo di marcatura troviamo traccia nell'opera di Salvator Angelo Spano (Villacidro 1925-2004), dove ddoi marca distanza fisica o temporale, figurando anche nel racconto dei ricordi di infanzia e nei contesti riportivi (cf. (59)), mentre la deissi spazio-temporale di (n) $c i$ è orientata sul parlante ('qui', 'ora'), oppure su un argomento topicale o saliente. Questa seconda proforma ricorre infatti più nel discorso diretto che nella narrazione (cf. (60)).

(59) a. Intrendu ddoi fiat unu pàtiu mannu e ariosu.

'Entrando c'era un cortile grande e spazioso.' [ricordo di infanzia]

b. Candu ddoi fiat su fogu allutu.

'Quando c'era il fuoco acceso.' [ricordo di infanzia]

c. Fiat perigulosu... e poi ca in pitzus ddoi fiant is fantàsimus.

'[Ci dicevano che] era pericoloso... e che in cima c'erano i fantasmi.'

(Spano, 2003, 26, 54, 30)

(60) a. Ecus innoi, signora mia!... nc'est una citatzioni de pretura.

'Ecco qui, signora! C'è una citazione in pretura.'

b. Certus predis murvonis chi ancora nci funti in giru.

'Certi preti musoni che ancora ci sono in giro.'

c. Po nosu no nci fiat spàssiu e vita prus bella de cussa.

'Per noi non c'era divertimento e vita più bella di quella.'

(Spano, 2003, 100, 122, 28) 
In conclusione, dai dati raccolti in tre varietà campidanesi (Sardara, Sanluri e Villacidro) emerge una chiara tendenza all'assolvimento di funzioni evidenziali con ddoi, ma in nessuna di queste varietà abbiamo trovato prova della dismissione dell'originaria funzione deittica spazio-temporale della stessa proforma.

\section{Effetti di definitezza, deissi, evidenzialità}

Nella discussione dell'alternanza di ESSE e HABERE (nelle varietà di Bono, Bonorva, Buddusò, Bitti, Orosei, e nella varietà $\mathrm{H}$ di Sardara e Sanluri) abbiamo notato che se, da un canto, la selezione dell'una o dell'altra copula sembrerebbe dipendere dalla definitezza del pivot (cf. (3) rispetto a (4) e (12) rispetto a (13)), dall'altro, si riscontrano delle devianze sistematiche da questo modello. HABERE non è mai selezionato con pivot esistenziali definiti, ma ESSE può essere scelto con classi di pivot indefiniti che coricorrono al livello interdialettale (cf. (6)-(8) e (14)-(16), (19)). Le classi in questione sono illustrate qui di seguito con dati di provenienza logudorese (Bono) e campidanese (Sardara - H).

(61) a. B'at / b'est un'isveglia chi funtzionat.

(Bono)

'C'è una sola sveglia che funziona bene.'

b. Cales alunnos bi sun / b'at?

(Bono)

'Quali alunni ci sono?'

c. Chie b'est? / b'at?

(Bono)

'Chi c'è?'

(62) a. (N)c'est ua femia sceti in sa vida mia.

(Sardara - H)

'C'è una sola donna nella mia vita.'

b. Cali funt is studenti chi (n)ci funt?

(Sardara - H)

'Quali studenti ci sono?'

c. Chi (n)c'est?

(Sardara - H)

'Chi c'è?'

d. No ddu est / (n)c'est nemus.

'Non c'è nessuno.'

$($ Sardara $-\mathrm{H})$

Il fatto che la distribuzione delle copule ESSE e HABERE sia solo in parte prevedibile dalla definitezza formale del pivot indica che, seppur codificati nella morfosintassi, gli effetti di definitezza del sardo non siano da questa stessa motivati. Lo studio di un più ampio campione di lingue sia romanze sia geneticamente non imparentate suggerisce, infatti, che gli effetti di definitezza codifichino vincoli di marcatezza semantica e pragmatica sul pivot e, in ultima analisi, sul soggetto (Bentley 2009). La correlazione fra questo genere di vincoli e gli effetti di definitezza è spiegata dall'ipotesi di Beaver/Francez/Levinson (2006) che i costrutti copulari canonici, cioè quelli in cui l'argomento soggetto precede il predicato, nelle lingue con ordine lineare SVO, siano in competizione con i costrutti esistenziali. Se all'unico argomento di un costrutto copulare mancano almeno alcune delle proprietà semantiche e pragmatiche dei soggetti canonici della lingua data, quest'argomento verrà di norma codificato come pivot di un costrutto esistenziale. I pivot esistenziali sono dunque soggetti non canonici, nel senso che non soddisfano, oppure soddisfano solo parzialmente, i vincoli di marcatezza che operano sul soggetto nella lingua data. Si veda il contrasto fra sos libros sun subra sa mesa (Bono) 'i libri sono sul tavolo', con soggetto canonico, e b'at libros subra sa mesa 'ci sono libri sul tavolo', il cui 
pivot non si qualificherebbe come soggetto di un costrutto copulare non esistenziale (*libros sun subra sa mesa, lit. libri sono sul tavolo).

Sebbene le proprietà dei soggetti canonici possano variare da lingua a lingua, in genere le lingue naturali evitano i soggetti indefiniti non generici (eccezion fatta per stili particolari che sono irrilevanti in questo contesto perché soggetti a convenzioni che annullano i vincoli operativi nei registri spontanei), dando preferenza ai soggetti topicali e identificabili (si veda sotto), altamente referenziali, agentivi e specifici (Comrie 1979, 19, Foley/Van Valin 1987, 115, Givón 1978, Jespersen 1924, 145-156, Keenan 1976). Queste restrizioni sono massimamente operative nei costrutti intransitivi attivi. L'ipotesi di Beaver/Francez/Levinson (2006) prevede, dunque, che di norma i pivot dei costrutti esistenziali siano focali, o non topicali, e scarsamente identificabili, referenziali, agentivi, specifici. ${ }^{19}$

I pivot dei costrutti esistenziali sardi codificano referenti focali o non topicali, cioè referenti che non fanno parte della presupposizione (Lambrecht 1994, 52). Basti notare che (63a) sarebbe un enunciato infelice nel contesto della domanda in (63b), proprio perché, in questo caso, il pivot apparterrebbe alla presupposizione. ${ }^{20}$

(63) a. B'est sorre tua in coghina.

'C'è tua sorella in cucina.'

(Bono)

b. In ube est sorre mea?

'Dov'è mia sorella?'

Lo studio delle varietà di Bono, Bonorva, Buddusò, Bitti, Orosei, Sardara $(\mathrm{H})$ e Sanluri $(\mathrm{H})$ indica che l'alternanza di ESSE e HABERE discrimina, in primo luogo, referenti identificabili da referenti non identificabili. Un referente è identificabile se il parlante può assumere che l'interlocutore ne abbia una rappresentazione cognitiva e, quindi, lo possa individuare nell'insieme di referenti che possono essere designati con la stessa espressione linguistica (Lambrecht 1994, 77-92). Ad esempio, il pivot degli esempi (3a-e) (Bi sun (cus)sos lib(e)ros 'ci sono (que)i libri') indica, nel contesto della domanda 'Cosa possiamo usare per fermare la porta?', non qualsiasi libro, ma un gruppo di libri che l'interlocutore può immediatamente identificare perché è presente nella sua memoria a lungo termine, o è già stato attivato nel contesto dell'enunciazione, oppure perché viene attivato dal deittico cussos 'quei'. ${ }^{21}$ Allo stesso modo, su postinu, negli esempi (13a-b), sia se si riferisce ad un ruolo, sia se si riferisce ad un individuo in particolare, ha una rappresentazione cognitiva per $\mathrm{i}$ locutori: 'il postino che serve questa zona'. I referenti identificabili sono di norma codificati da pivot formalmente definiti.

\footnotetext{
${ }^{19}$ La discussione delle diverse manifestazioni degli effetti di definitezza che si riscontrano nelle lingue naturali, e la loro interpretazione alla luce dell'ipotesi degli studiosi menzionati sopra, andrebbe al di là degli intenti di questo lavoro. Si veda Bentley (2009) per un'analisi del contrasto fra (i) le lingue che tendono a non licenziare pivot formalmente definiti (per esempio, il francese, dove un costrutto come il y a moi è grammaticale solo nella lettura a lista, discussa in Abbott 1993 e Rando/Napoli 1978), (ii) le lingue con marcatura differenziale dei pivot (come le varietà sarde discusse sopra) e, infine, (iii) le lingue che sembrano ammettere pivot definiti senza marcarli diversamente da quelli indefiniti (si vedano l'italiano e il rumeno). Nella stessa sede si discutono anche gli effetti di definitezza di lingue topic-prominent (per esempio, il cinese mandarino), che, almeno secondo certe teorie linguistiche, non hanno una funzione sintattica di soggetto.

20 In costrutti come su dinari b'est 'i soldi ci sono', l'argomento topicale è licenziato dal predicato in focus contrastivo. Codificato in una posizione precopulare, questo argomento va analizzato come il tema di un costrutto copulare locativo, e non come il pivot di un costrutto esistenziale.

${ }^{21}$ Un concetto è attivo se è parte di ciò a cui si presta attenzione in un particolare momento dell'enunciazione (Van Valin/LaPolla 1997, 200).
} 
In Bentley (2004) è stato notato che i referenti non identificabili possono essere codificati come pivot di costrutti esistenziali con la copula ESSE quando sono ancorati, cioè esplicitamente messi in relazione ad un altro referente o a una cornice concettuale identificabile (Lambrecht 1994, 77-92, Prince 1981). La scelta di HABERE, invece, indica che il referente in questione non solo non è identificabile, ma non è neanche ancorato.

(64) a. Bi sun medas fiores in sa tanca e atteros in su ortu.

(Bono)

'Ci sono molti fiori nel campo, altri nell'orto.'

b. B'at medas fiores in sa tanca.

'Ci sono molti fiori nel campo.'

È interessante notare che il pivot di (64a) potrebbe anche essere ammesso come soggetto di un costrutto copulare canonico ((?)Medas fiores sun in sa tanca e atteros in su ortu), ma quello di (64b) non potrebbe figurare in questo tipo di costrutto (*Medas fiores sun in sa tanca, dove medas fiores non è contrastivo).

I dati raccolti in questo studio suggeriscono che la selezione di ESSE è inoltre sensibile al parametro della specificità, visto che i pivot degli esempi in (6), (7), (14), (15), (61a-b) e (62a-b) sono referenziali e unici o partitivi. Per quanto riguarda il parametro della referenzialità, si veda anche la diversa scelta della copula in (65a) e (65b).

(65) a. B'at un'ómine in sa ianna.

(Bono)

'C'è uno / un uomo alla porta.'

b. Unu máttulu de fiores chi prima non (n)che fin.

'Un mazzo di fiori che prima qui non c'erano.'

(Bono)

Mentre il pivot di (65a), un'ómine, richiede una lettura non specifica, quello di (65b), unu máttulu de fiores, è referenziale e, quindi, specifico (Fodor/Sag 1982). Ancora una volta, val la pena di notare che il pivot di (65a), con lettura non specifica, non si presterebbe alla codifica preverbale in un costrutto copulare canonico (*?Un'ómine est in sa ianna).

Per quanto riguarda la lettura partitiva (Enç 1991), si noti che il pivot di (61b) (Cales alunnos bi sun?) e (62b) (Cali funt is studenti chi (n)ci funt?) indica un sottoinsieme di specifiche classi di studenti. L'informatrice che ha fornito il dato in (61b) ha infatti chiarito spontaneamente che l'esempio si riferisce ad una classe di studenti che si conosce, mentre per riferirsi genericamente a qualsiasi studente si sceglierebbe la forma Cales alunnos b'at?. La variazione illustrata in $(61 b) /(7 b)$ non è dunque libera.

I costrutti con il pivot cales 'quali' contrastano, quanto alla selezione della copula, con quelli con cantos 'quanti' (cf. (7), (9), (15), (17)). Anche questo dato, inspiegabile dal punto di vista strettamente morfosintattico, si chiarisce alla luce dell'ipotesi che $\mathrm{i}$ pivot esistenziali siano cattivi candidati per la funzione sintattica soggetto. Infatti, cantos 'quanti', diversamente da cales 'quali', non comporta una presupposizione di esistenza e quindi non si presta alla lettura partitiva o specifica di cui sopra. ${ }^{22}$ Cantos è dunque un cattivo soggetto, e viene marcato di conseguenza. Anche in altre lingue 'quanto', a differenza di 'quale', può non comportarsi da soggetto canonico. Sono significative, in tal senso, sia la discussione di Suñer (1992, 660-661) sul mancato

\footnotetext{
${ }^{22}$ Sulla quantificazione forte, prevista da which, e debole, prevista da how many, si veda Heim (1987).
} 
accordo del presunto soggetto con il verbo e il clitico soggetto in fiorentino e in trentino sia la testimonianza dei costrutti esistenziali spagnoli, parallela a quella degli esistenziali sardi (Belloro 2007, 90).

Infine, dalla testimonianza di dati come quelli in (61c), (62c-d), (8a-c, e) e (16a-b) (che contrastano con (10a-e) e (18a)) deduciamo che la selezione della copula possa dipendere dal parametro dell'animatezza. A questo proposito, si noti anche che i pivot esistenziali delle varietà sarde in questione possono essere pronomi personali e che questi richiedono la scelta di ESSE.

(66) a. A bi ses (tue) stasera?

(Bono)

'Ci sarai (tu) stasera?

b. No ti preoccùpisti: (n)ci seu deu.

'Non ti preoccupare: ci sono io.'

(Sardara - H)

Com'è noto, i pronomi personali occupano una posizione molto alta nella gerarchia di animatezza, prestandosi così alla funzione di soggetto e richiedendo la marcatura differenziale, se in funzione di oggetto, in una serie di lingue accusativo / nominative (Bossong 1991, Floricic 2003, Jones 1993, Sornicola 1997, etc.). I dati in (66a-b) mostrano che, se si riferiscono a referenti non topicali, i pronomi possono essere codificati come pivot esistenziali, ma in questo caso richiedono la copula di default, ESSE, e controllano l'accordo della stessa, al pari dei soggetti dei costrutti copulari canonici.

Poiché le proprietà pragmatiche (identificabilità) e semantiche (specificità, animatezza) determinanti per la selezione della copula corrispondono a parametri pertinenti alla selezione e la codifica del soggetto, in sardo come in altre lingue naturali, i nostri dati confortano l'ipotesi che gli effetti di definitezza dipendano in ultima analisi da vincoli di marcatezza sul soggetto. ${ }^{23}$

Proponiamo dunque che l'alternanza delle copule dei costrutti esisteziali sardi è parte di un sistema di marcatura differenziale dei soggetti. Nelle varietà qui discusse, $i$ soggetti topicali vengono di norma codificati nella posizione immediatamente preverbale o precopulare, richiedono la copula o l'ausiliare perfettivo di default, e controllano l'accordo su tale forma verbale. ${ }^{24}$

a. Sorre tua est in coghina.

(Bono)

'Tua sorella è in cucina.'

b. Sas pitzinnas sun bennidas.

(Bono)

'Le ragazze sono venute.'

(68) a. Sa scola est in pratza 'e mercau.

$($ Sardara - H)

'La scuola è nella piazza del mercato.'

b. Is librus funt arribaus.

(Sardara - H)

'I libri sono arrivati.'

\footnotetext{
${ }^{23}$ Invece dell'agentività, il criterio che sembra pertinente alla codifica del pivot, nelle nostre varietà, è il tratto più alto del parametro dell'animatezza (+ umano). Animatezza e agentività sono d'altronde parametri collegati, nel senso che il secondo non può prescindere dal primo.

${ }^{24}$ Per gli ausiliari perfettivi in sardo, si vedano Jones (1988) e Lepori (2001, 59-69).
} 
I soggetti non topicali vengono di norma codificati in posizione postcopulare o postverbale. $^{25}$

(69) a. B'est sorre tua in coghina.

(Bono)

'C'è tua sorella in cucina.'

b. (Bi) sun bennidas sas pitzinnas.

(Bono)

'Sono venute le ragazze.'

(70) a. In pratza 'e mercau ddu est sa scola.

(Sardara - H)

'Nella piazza del mercato c’è la scuola.'

(Sardara - H)

b. Funt arribaus is librus.

'Sono arrivati i libri.'

Pur essendo marcati come non canonici dalla posizione sintattica, questi argomenti si comportano come soggetti, essendo controllori dell'accordo verbale sulla copula o l'ausiliare perfettivo di default.

La violazione degli altri vincoli di marcatezza sul soggetto può essere marcata dalla scelta della copula o l'ausiliare perfettivo non canonici e dall'assenza dell'accordo sulla forma verbale finita, cioè da codifiche morfosintattiche diverse da quelle che individuano il soggetto. ${ }^{26}$

(71) a. B'at fiores subra sa mesa.

(Bono)

'Ci sono fiori sul tavolo.'

b. B'at bennidu pitzinnas.

'Sono venute ragazze.'

(Bono)

(72) a. No ddu at scolas.

$($ Sardara $-\mathrm{H})$

'Non ci sono scuole.'

b. Nd'at arribaus troppus, de librus.

'Ne sono arrivati troppi, di libri.'

(Sardara - H)

Gli effetti di definitezza delle varietà sotto esame, pertanto, fanno parte di un sistema di codifica morfosintattica che distingue i soggetti canonici (cf. (67) e (68)) da quelli non-canonici (cf. (69) e (70)) e dagli argomenti non eligibili alla funzione sintattica di soggetto (cf. (71) e (72)). ${ }^{27}$

Un ulteriore indizio a favore dell'ipotesi che gli effetti di definitezza, seppur codificati nella morfosintassi, dipendano da vincoli di marcatezza semantici e pragmatici proviene dai dati illustrati qui di seguito.

\footnotetext{
${ }^{25}$ Eccezion fatta per la costruzione nota come focus fronting (irrilevante in questo contesto perché codifica della forza illocutiva interrogativa o di focus contrastivo), che non prevede comunque che il pivot o il soggetto occupino la posizione canonica del soggetto (Jones 1993, Mensching/Remberger in stampa).

${ }^{26}$ I dati logudoresi e campidanesi in $(71 b)$ e $(72 b)$ contrastano dal punto di vista dell'accordo verbale sulla forma non finita del verbo. In questa sede non ci soffermeremo su questo punto, visto che l'accordo del participio passato non è, nelle lingue sotto esame, un criterio diagnostico che identifica tutte le possibili classi di soggetto (Loporcaro 1998, Bentley 2006, 236).

${ }^{27}$ Questa proposta, ispirandosi all'ipotesi impersonale degli effetti di definitezza (Belletti 1988, La Fauci/Loporcaro 1997, Moro 1997, Perlmutter 1983, etc.), la rivisita dal punto di vista dell'interfaccia della semantica, la pragmatica e la sintassi. Per gli aspetti formali della nostra analisi degli effetti di definitezza, rimandiamo a Bentley (2009), dove esaminiamo lingue non necessariamente imparentate.
} 
(73) a. Tzertas iscolas chi che sun in bidda.

(Bono)

b. Tzertas iscolas chi b'at in Buddusò.

(Bono)

'Certe scuole che ci sono qui in paese / a Buddusò.'

(74) a. Tzertas iscolas chi che sun in bidda.

(Bonorva)

b. Tzertas iscolas chi b'at in Bono.

(Bonorva)

'Certe scuole che ci sono qui in paese / a Bono.'

(75) a. Tzertas iscolas chi che son in bidda.

(Buddusò)

b. Tzertas iscolas chi b'at in Utieri.

(Buddusò)

'Certe scuole che ci sono qui in paese / a Ozieri.'

Oltre all'alternanza canonica di (n)che e bi, gli esempi in (73)-(75) sono caratterizzati dalla coricorrenza di ESSE con la prima proforma e HABERE con l'altra. Sebbene la scelta di ESSE e HABERE non sembri a prima vista dipendere dalla variazione del pivot, il fatto che lo stesso risultato emerga indipendentemente in tre delle varietà sondate, quelle logudoresi, induce a pensare che l'alternanza delle copule non sia un semplice errore di esecuzione. Piuttosto la covariazione delle copule e delle proforme si spiega se il quantificatore del pivot (tzertas 'certe') riceve una lettura specifica o forte nei costrutti in cui la proforma indica prossimità. Se questa ipotesi è valida, la selezione della proforma e quella della copula non sono ortogonali, ma interagiscono nella misura in cui la deissi del costrutto esistenziale contribuisce all'interpretazione della semantica del pivot. Si osservi che, se il pivot è accompagnato da un quantificatore universale (e ai nostri scopi tale può ritenersi l'articolo definito), la copula non covaria con la proforma.

(76) a. Sas iscolas chi che sun in bidda.

(Bono)

b. Sas iscolas chi bi sun in Buddusò.

(Bono)

'Le scuole che ci sono qui in paese / a Buddusò.'

(77) a. Sas iscolas chi che sun in bidda.

(Bonorva)

b. Sas iscolas chi bi sun in Bono.

(Bonorva)

'Le scuole che ci sono qui in paese / a Bono.'

(78) a. Sas iscolas chi che son in bidda.

(Buddusò)

b. Sas iscolas chi bi son in Utieri.

(Buddusò)

'Le scuole che ci sono qui in paese / a Ozieri.'

Il fatto che lo stesso tipo di covariazione non sia stato rilevato nei dati di Orosei, Bitti, Sardara e Sanluri, cioè le altre varietà con l'alternanza della copula, dipenderà, almeno in qualche misura, dalla mancanza di un'alternanza altrettanto sistematica di due proforme. Come si spiegava nella sezione 3, infatti, l'unica proforma riscontrata ad Orosei, e nella varietà L di Bitti, è $b i$.

(79) Carchi / Calicuna iscola chi b'est inoche / in cue / incuddaiche. (Orosei)

'Qualche scuola che c'è qui / lì / lì (ancora più lontano).'

(80) Carchi iscola chi bi son in Bithi / in cue.

'Qualche scuola che c'è qui a Bitti / lì.'

(Bitti - L) 
Nelle varietà arcaiche di Sardara e Sanluri, oltre alle proforme dettiche $(n) c i$ e $d d o i$, è usata la proforma neutra $d d u$ e questa potrebbe essere la causa dell'assenza, nel nostro corpus, di dati probanti in questo contesto.

Se la selezione della proforma interagisce con quella della copula, limitatamente al caso esposto sopra, la deissi delle proforme è senza dubbio all'origine della marcatura evidenziale che abbiamo rilevato nei costrutti esistenziali delle varietà campidanesi di Sardara, Sanluri e Villacidro. Ricapitoliamo innanzitutto i risultati riguardanti la deissi delle proforme. Nelle sezione 3 si è notato che le varietà logudoresi e la varietà $H$ del bittese alternano (n)che e bi. La prima di queste due proforme è il termine marcato dell'opposizione deittica in questione, nel senso che le inferenze deittiche che derivano dalla scelta di questa proforma non sono cancellabili, al contrario di quelle che derivano dalla selezione di $b i$ (si veda la nota 12 ). Nelle varietà logudoresi e nel bittese più conservativo, dunque, la forma marcata è quella collegata positivamente al centro di orientamento costituito dal parlante. La non distruttibilità delle inferenze deittiche che derivano dall'uso di questa proforma si riflette nell'interpretazione specifica dei quantificatori che ammettono letture forti e deboli (cf. (73)-(75)).

Nelle varietà campidanesi con l'alternanza di due proforme, invece, il termine marcato ddui/ddoi è quello definito negativamente rispetto al parlante, mentre $(n) c i$ può indicare coincidenza, inclusione o prossimità alla locazione del parlante, ma può anche essere una forma neutra. Non a caso, in alcune di queste varietà la forma marcata $d d o i / d d u i$ non si oppone a $(n) c i$ soltanto in termini spazio-temporali, ma anche nel connotare il contenuto proposizionale del costrutto dal punto di vista della fonte dell'informazione data. Nella varietà più conservativa di Sardara e Sanluri la selezione di ddoi / ddui marca la non testimonialità o l'assenza di un'esperienza visiva concomitante con l'enunciazione (cf. (24a-b), (25b), (26b), (54a), (55a), (56a-b), $(57 \mathrm{a}-\mathrm{b}))$. Nella varietà $\mathrm{H}$ di Villacidro la scelta di ddoi distingue i ricordi e le dicerie dall'esperienza presente e di prima mano (cf. (27b), (28b), (29b), (30b), (58a-c) e $(59 \mathrm{a}-\mathrm{c}))$.

Il fatto che le connotazioni evidenziali di $d d o i / d d u i$ siano definibili in termini negativi (la non testimonialità, l'assenza di un'esperienza visiva dell'esistente, la riportività, etc.) si spiega alla luce della deissi spaziale di queste proforme, che, secondo il modello d'analisi proposto da Vanelli (1972) per l'italiano, è definita negativamente rispetto al parlante. Allo stesso tempo, non sorprende che siano proprio i costrutti campidanesi a licenziare connotazioni evidenziali, visto che le funzioni evidenziali definite negativamente rispetto al parlante sono fra le più attestate nelle lingue naturali. Basti pensare alla constatazione di De Haan $(2005,314)$ che, nelle lingue con sistemi di marcatura dell'evidenzialità, le informazioni riportate sono marcate più frequentemente e sistematicamente di quelle di prima mano (si veda anche Aikhenvald 2004, 1).

Con riferimento alla marcatura evidenziale dei costrutti esistenziali di Sardara Sanluri e Villacidro bisogna, a questo punto, stabilire se questa vada considerata un vero e proprio sistema di marcatura grammaticale dell'evidenzialità. In secondo luogo, è opportuno collocare i dati rilevati nel contesto dell'espressione dell'evidenzialità nelle lingue romanze.

Com'è noto, soltanto alcune lingue grammaticalizzano l'evidenzialità, altre la grammaticalizzano solo parzialmente, e altre ancora si limitano ad usare mezzi lessicali per esprimerla (Ramat 1996, 290-291, Squartini 2005). Secondo Aikhenvald (2004, 38-39, 105-151) quest'ultimo tipo di lingua si serve di strategie evidenziali, cioè estensioni semantiche di forme non evidenziali, piuttosto che di un vero e proprio sistema grammaticale evidenziale. La gran parte delle lingue romanze appartiene a 
questo tipo, visto che esprime l'evidenzialità con morfemi flessivi, come quelli del futuro e del condizionale, la cui funzione primaria è un'altra (si veda Squartini $2001 \mathrm{e}$ i riferimenti ivi citati). Un'eccezione a questa tendenza è discussa in Squartini (2005), dove, in base alla corrispondenza biunivoca forma-funzione evidenziale, si dimostra che il presuntivo rumeno è una forma che grammaticalizza l'evidenzialità. ${ }^{28}$

Dai dati riportati in questo studio risulta chiaro che le proforme deittiche dei costrutti esistenziali delle varietà campidanesi qui discusse non costituiscono un sistema formale che grammaticalizza l'evidenzialità, e ciò almeno per tre motivi. In primo luogo, l'opposizione $(n) c i \sim d d o i / d d u i$ ha altre funzioni, chiaramente non evidenziali, relative alla marcatura della deissi spazio-temporale degli esistenziali campidanesi. In secondo luogo, la marcatura evidenziale dei costrutti esistenziali non è obbligatoria, mentre nelle lingue con sistemi evidenziali grammaticali, l'omissione della marcatura evidenziale è causa di agrammaticalità. Infine, non ci risulta che le funzioni evidenziali di $d d o i / d d u i$ si estendano al di là dei costrutti esistenziali, cioè che ddoi / ddui sia la marca dell'evidenzialità nelle varietà in questione. D'altro canto, come spiega Aikhenvald $(2004,105)$, una lingua può usare strategie evidenziali diverse in costrutti diversi. Si noti, tuttavia, che la funzione evidenziale di ddoi / ddui nei costrutti esistenziali può marginalmente prescindere dalla deissi degli stessi, come in (81) (cf. (24b)), che non implica necessariamente distanza dal parlante.

$$
\begin{aligned}
& \text { Chi ddoi at? } \\
& \text { 'Chi c'è?' [contesto: non si vede nessuno] }
\end{aligned}
$$

(Sardara - L)

La funzione evidenziale di $d d o i / d d u i$ è dunque una strategia, cioè un'estensione semantica di un'altra funzione, ma sembrerebbe essere in via di convenzionalizzazione, per lo meno nel contesto dei costrutti esistenziali. Concludiamo che i costrutti esistenziali campidanesi offrono una testimonianza chiara di lessicalizzazione, ma non di grammaticalizzazione, dell'evidenzialità.

Che la deissi delle lingue naturali sia fonte di strategie evidenziali è d'altro canto un fatto riconosciuto in letteratura. Aikhenvald (2004, 130-131, 275-276) discute l'uso dei dimostrativi per marcare la visibilità o meno di un oggetto, notando che la visibilità è spesso associata alla vicinanza ai parlanti. In diacronia, le marche direzionali e locative vengono talvolta grammaticalizzate in veri e propri sistemi evidenziali. Nel contesto romanzo è particolarmente significativo che le nostre varietà sfruttino le proforme deittiche per l'espressione dell'evidenzialità, visto che, nelle lingue sorelle, il sistema verbale è l'unica fonte riconosciuta di strategie o sistemi evidenziali (con la possibile eccezione dei marcatori enunciativi occitani discussi in Pusch 2008). Se la tendenza alla generalizzazione di nci arrivasse ad eliminare la controparte deittica $d d o i$ / ddui, il dominio funzionale di questa forma potrebbe di conseguenza restringersi ad una funzione prettamente evidenziale. Ma di questo eventuale sviluppo non abbiamo al momento alcun indizio, a parte l'emergere marginale di esempi come (81).

\section{Conclusioni}

\footnotetext{
${ }^{28}$ Più precisamente questa forma rumena ha funzioni esclusivamente evidenziali se l'inferenzialità si ritiene una funzione evidenziale, al pari di quella riportiva (Squartini 2005).
} 
In questo lavoro abbiamo riportato i risultati di un'indagine sui costrutti esistenziali delle varietà sarde parlate a Bono (logudorese sud-orientale, si veda Virdis 1988), Bonorva e Buddusò (logudorese centrale), Bitti e Orosei (nuorese settentrionale), Orgosolo e Fonni (nuorese orientale e meridionale), Terralba, Sardara, Sanluri, Villacidro e Monastir (campidanese centro-occidentale), ponendo particolare attenzione alla distribuzione diatopica e funzionale delle copule (logudorese e nuorese éssere e áere, campidanese essi e ai) e delle proforme esistenziali (logudorese e

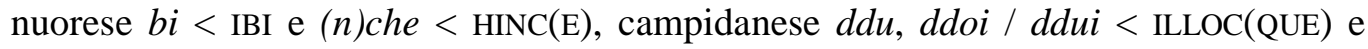

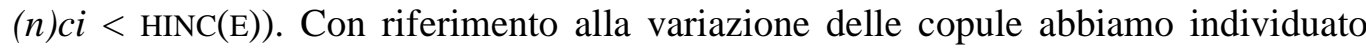
un'area sostanzialmente omogenea, che comprende il logudorese centrale e sudorientale e il nuorese settentrionale, mentre le rimanenti aree si presentano relativamente disomogenee, a causa di fenomeni divergenti di generalizzazione di copule diverse. È interessante che varietà affini dal punto di vista fonetico, per esempio quelle di Fonni e Orgosolo, entrambe appartenenti alla Barbagia di Ollolài (o gruppo Fonni), abbiano generalizzato copule esistenziali diverse (éssere a Fonni e áere ad Orgosolo). Da questo punto di vista, i nostri dati confortano l'osservazione di Wolf $(2007,116)$ che la zona in questione non manca mai di riservare sorprese al ricercatore.

A differenza della maggior parte delle varietà campidanesi, che ha generalizzato la copula essi, le varietà dei non giovani di Sardara, Sanluri e, con modalità diverse, Villacidro, presentano ancora l'alternanza di essi e ai. Questi punti si sono dunque rivelati i più conservativi nell'area campidanese da noi sondata.

Dal punto di vista funzionale, l'alternanza delle copule codifica effetti di definitezza affini a quelli già discussi in letteratura. Tuttavia, il fatto che la selezione della copula non sia interamente prevedibile dalla definitezza morfosintattica del pivot indica che, seppur manifestati nella morfosintassi, i suddetti effetti siano motivati da fattori semantici e la pragmatici. Svelando correlazioni sinora inosservate fra la selezione di éssere/essi e la specificità o l'animatezza del pivot, i risultati del nostro sondaggio corroborano l'ipotesi di Beaver/Francez/Levinson (2006) che gli effetti di definitezza dipendano in ultima analisis da vincoli di marcatezza sul soggetto. In area campidanese (Villacidro e, marginalmente, Sardara) la scelta di ai può anche marcare il contenuto proposizionale del costrutto esistenziale dal punto di vista della fonte dell'informazione data.

Con riferimento alle proforme, l'area nuorese si è rivelata fratta anche nell'ambito della sottovarietà settentrionale, poiché la testimonianza del bittese è assimilabile a quella del logudorese centrale e sud-orientale, distinguendosi da quella delle rimanenti varietà. Infatti, a Bitti, così come a Bono, Bonorva e Buddusò, abbiamo riscontrato l'alternanza di due proforme ( $b i<\mathrm{IBI}$ e $(n)$ che < HINC(E)), l'una delle quali, $(n)$ che, ha le caratteristiche del termine marcato delle opposizioni binarie di verbi deittici di movimento (Ricca 1993). L'alternanza di due proforme (ddoi / ddui < ILLOC(QUE) e $(n) c i<\operatorname{HINC}(\mathrm{E}))$ si riscontra anche in alcune varietà campidanesi, dove, però, la forma marcata $d d o i / d d u i$ è quella definita negativamente rispetto al centro deittico dell'enunciazione costituito dal parlante. Se, in logudorese, la non distruttibilità delle inferenze deittiche che derivano dall'uso della proforma marcata (n)che si riflette nell'interpretazione specifica dei quantificatori del pivot che ammettono letture forti (Milsark 1979), in area campidanese, la scarsa cancellabilità delle inferenze deittiche che derivano dalla selezione di ddoi / ddui, termine deittico definito negativamente rispetto al centro dell'enunciazione, ha dato luogo all'insorgere di un sistema di marcatura evidenziale che codifica la non testimonialità e l'assenza di un'esperienza visiva dell'esistente (Sardara, Sanluri) o distingue 
l'esperienza presente e di prima mano dai sogni, i ricordi e le dicerie (Villacidro). Dall'analisi dei dati risulta tuttavia chiaro che l'alternanza delle proforme esistenziali delle varietà campidanesi sotto esame non è un sistema formale che grammaticalizza l'evidenzialità. La funzione evidenziale di ddoi / ddui ha, piuttosto, chiare caratteristiche di una strategia lessicale in via di convenzionalizzazione.

\section{Riferimenti bibliografici}

\section{Fonti primarie}

Delogu, Ignazio (ed.), Il condaghe di San Pietro Silki. Testo logudorese inedito dei secoli XI-XIII, Sassari, Dessì, 1997.

Pintore, Gianfranco, Morte de unu Presidente, Cagliari, Condaghes, 2007.

Spano, Salvator Angelo, Genti mia, Sassari, Editrice Democratica Sarda, 2003.

Solmi, Arrigo (ed.), Le carte volgari dell'archivio arcivescovile di Cagliari. Testi campidanesi dei secoli XI-XIII, Firenze, Tipografia Galileiana, 1905.

\section{Fonti secondarie}

Abbot, Barbara, A pragmatic account of the definiteness effect in existential sentences, JoP 19 (1993), 39-55.

Aikhenvald, Alexandra, Evidentiality, Oxford, Oxford University Press, 2004.

Beaver, David/Francez, Itamar/Levinson, Dmitry, Bad subject: (non-) canonicality and NP distribution in existentials, in: Georgala, Effi/Howell, Jonathan (edd.), Proceedings of Semantic and Linguistic Theory XV, 25-27 March 2005, Ithaca, N.Y, Cornell University Press, 2006, 19-43.

Belletti, Adiana, The case of unaccusatives. LingI 19 (1988), 1-34.

Belloro, Valeria, Spanish Clitic Doubling. A Study of the Syntax-Pragmatics Interface, tesi di dottorato, State University of New York at Buffalo, 2007.

Bentley, Delia, Definiteness effects: evidence from Sardinian, TPhS 102 (2004), 57101.

Bentley, Delia, Split intransitivity in Italian (EALT 30), Berlin, Mouton de Gruyter, 2006.

Bentley, Delia, Harmonic alignment and the definiteness effects of existential constructions, Ms., University of Manchester, 2009.

Blasco Ferrer, Eduardo, La lingua sarda contemporanea. Grammatica del logudorese $e$ del campidanese. Norma e varietà dell'uso. Sintesi storica, Cagliari, Della Torre, 1986.

Blasco Ferrer, Eduardo, Le parlate dell'Alta Ogliastra. Analisi dialettologica. Saggio di storia linguistica e culturale, Cagliari, Della Torre, 1988.

Blasco Ferrer, Eduardo, Crestomazia sarda dei primi secoli. Volume primo. Testi. Grammatica storica. Glossario (Officina Linguistica 4), Nuoro, Ilisso, 2003.

Bossong, Georg, Differential object marking in Romance and beyond, in: Wanner, Dieter/Kibbee, Douglas A. (edd.), New analyses in Romance linguistics (Current Issues in Linguistic Theory 69), Amsterdam / Philadelphia, Benjamins, 1991, 143 170.

Comrie, Bernard, Definite and animate direct objects: a natural class, Linguistica Silesiana 3 (1979), 13-21.

Contini, Michel, Étude de géographie phonétique et de phonétique instrumentale du sarde, Alessandria, Edizioni Dell'Orso, 1987. 
Corda, Francesco, Grammatica moderna del sardo logudorese, Cagliari, Della Torre, 1994.

De Haan, Ferdinand, Semantic distinctions of evidentiality, in: Haspelmath, Martin/Dryer, Matthew/Gil, David/Comrie, Bernard (edd.), World Atlas of Language Structures, Berlin / New York, Mouton de Gruyter, 2005, 314-317.

Enç, Mürvet, The semantics of specificity. LingI 22 (1991), 1-25.

Fillmore, Charles, Santa Cruz lectures on deixis 1971, Bloomington, IULC, 1975.

Floricic, Franck, Notes sur l'accusatif prépositionnel en sarde, BSL(P) 98 (2003), 247-303.

Fodor, Jerry/Sag, Ivan Referential and Quantificational Indefinites, Linguistics and Philosophy 5 (1982), 355-398.

Foley, William/ Van Valin, Robert Jr, Functional syntax and universal grammar, Cambridge, Cambridge University Press, 1987.

Givón, Talmy, Definiteness and referentiality, in: Greenberg, Joseph H. (ed.), Universals of human language. Volume 4. Syntax, Stanford, Stanford University Press, 1978, 291-330.

Heim, Irene, Where does the definiteness restriction apply? Evidence from the definiteness of variables, in: Reuland, Eric J./ter Meulen, Alice G. B. (edd.), The Representation of (In)definiteness, Cambridge, Massachussets, MIT Press, 1987, 21-42.

Huang, Yan, Pragmatics, Oxford, Oxford University Press.

Jespersen, Otto, The philosophy of grammar. London, George Allen and Unwin, LTD, 1924.

Jones, Michael A. Auxiliary verbs in Sardinian. Transactions of the Philological Society 86 (1988), 173-203.

Jones, Michael A. Sardinian syntax, London, Routledge, 1993.

Jones, Michael A. The prepositional accusative in Sardinian: its distribution and syntactic repercussions, in: Maiden, Martin/Smith, John Charles (edd.), Linguistic Theory and the Romance Languages (Current Issues in Linguistic Theory 122), Amsterdam / Philadelphia, Benjamins, 37-75.

Keenan, Edward L., Towards a universal definition of "subject", in: Li, Charles N. (ed.), Subject and Topic, New York / San Francisco / London, Academic Press, Inc., 1976, 303-333.

La Fauci, Nunzio/Loporcaro, Michele, Grammatical relations and syntactic levels in Bonorvese morphosyntax, in: Belletti, Adriana (ed.), Syntactic theory and the dialects of Italy, Torino, Rosenberg \& Sellier, 1993, 155-203.

La Fauci, Nunzio/Loporcaro, Michele, Outline of a theory of existentials on evidence from Romance, Studi italiani di linguistica teorica e applicata 26 (1997), 5-55.

Lambrecht, Knud, Information Structure and Sentence Form, Cambridge, Cambridge University Press, 1994.

Lepori, Antonio, Gramàtica sarda po is campidanesus. Duas obras in d-unu libru, Cuartu Sant'Aleni, Imprentas C.R., 2001.

Loporcaro, Michele, Sintassi comparata dell'accordo participiale romanzo, Torino, Rosenberg \& Sellier, 1998.

Mensching, Guido/Remberger, Eva-Maria, The left periphery in Sardinian, in: D'Alessandro, Roberta/Ledgeway, Adam/Roberts, Ian (edd.), The syntax of Italian dialects, Cambridge, Cambridge University Press, in stampa.

Meyer Lübke, Wilhelm, Romanisches Etymologisches Wörterbuch, Heidelberg, Winter, 1911.

Migliorini, Bruno, Storia della lingua italiana, Firenze, Sansoni, 1960. 
Milsark, Gary, Existential Sentences in English, tesi di dottorato, Massachusetts Institute of Technology, 1974.

Milsark, Gary, Existential Sentences in English, New York / London, Garland, 1979.

Moro, Andrea, The Raising of Predicates, Cambridge, Cambridge University Press, 1997.

Paulis, Giulio, Falsi diplomatici: il caso delle Carte Volgari dell'archivio arcivescovile cagliaritano, in: Paulis, Giulio (ed.), 1997a, 133-139.

Paulis, Giulio, Linguistica e filologia delle Carte Volgari dell'archivio arcivescovile cagliaritano, in: Paulis, Giulio (ed.), 1997b, 141-143.

Paulis, Giulio, Un ghost-word del sardo medioevale, in: Paulis, Giulio (ed.), 1997c, 145-148.

Paulis, Giulio (ed.), Studi sul sardo medioevale (Officina Linguistica 1), Nuoro, Ilisso, 1997.

Perlmutter, David, Personal and impersonal constructions, NLLT 1 (1983),141-200.

Pittau, Massimo, Pronunzia e scrittura del sardo logudorese, Sassari, Libreria Dessì, 1978.

Prince, Ellen, Toward a taxonomy of given-new information, in: Cole, Peter (ed.), Radical Pragmatics, New York / London, Academic Press, 1981, 223-255.

Pusch, Claus, Is there any evidence for evidentiality in Gascony Occitan?, Italian Journal of Linguistics 19 (2007), 91-108.

Ramat, Paolo, «Allegedly John is ill again»: stratégies pour le médiatif, in: Guentchéva, Zlatka (ed.), L'énonciation médiatisée, Louvain/Paris, Peeters, 1996, 287-298.

Rando, Emily/Napoli, Donna Jo, Definites in there-sentences. Language 54 (1978), 300-313.

Remberger, Eva-Maria, Null subjects, expletives and locatives in Sardinian, in: Kaiser, Georg,/Remberger, Eva-Maria (edd.), Proceedings of the workshop "Nulls subjects, expletives and locatives in Romance”, Konstanz, Fachbereich Wisssenschaft der Universität Konstanz, 2009, 231-261.

Ricca, Davide, I verbi deittici di movimento in Europa: una ricerca interlinguistica, Firenze, La Nuova Italia Editrice, 1993.

Sornicola, Rosanna, L'oggetto preposizionale in siciliano antico $e$ in napoletano antico. Considerazioni su un problema di tipologia diacronica, ItSt 18 (1997), 4559.

Squartini, Mario, The internal structure of evidentiality in Romance, StLa 25 (2001), 297-334.

Squartini, Mario, L'evidenzialità in rumeno e nelle altre lingue romanze, ZrP 121 (2005), 246-268.

Suñer, Margarita, Subject clitics in the Northern Italian vernaculars and the matching hypothesis, NLLT 10 (1992), 641-672.

Vanelli, Laura, La deissi in italiano, Padova, UNIPRESS, 1972.

Van Valin, Robert Jr./LaPolla, Randy, Syntax. Structure, meaning and function, Cambridge, Cambridge University Press, 1997.

Virdis, Maurizio, Sardisch: Areallinguistik, in: Holtus, Günter/Metzeltin, Michael/Schmitt, Christian (edd.), Lexikon der romanistischen Linguistik. Band 4: Italienisch, Korsisch, Sardisch, Tübingen, Max Niemeyer Verlag, 1988, 897-913.

Virdis, Maurizio, Tipologia e collocazione del sardo tra le lingue romanze, Ianua. Revista Philologica Romanica 4 (2003), http://www.romaniaminor.net/ianua. 
Wagner, Max L., Lautlehre des südsardischen Mundarten mit besonderer Berücksichtigung der um den Gennargentu gesprochenen Varietäten (Beihefte zur Zeitschrift für romanische Philologie 12), Halle, Max Niemeyer Verlag, 1907.

Wagner, Max L., Dizionario etimologico sardo. Vol. 1, Heidelberg, Winter, 1960.

Wagner, Max L., Historische Lautlehre des Sardischen (Beihefte zur Zeitschrift für romanische Philologie 93), Halle, Max Niemeyer Verlag, 1941.

Wolf, Heinz. J., Knacklaut in Orgosolo, ZrP 101 (1985), 267-311.

Wolf , Heinz. J., Sard. muniśa “balai”, RliR 71 (2007), 111-118.

Zamboni, Alberto, Alle origini dell'italiano. Dinamiche e tipologie della transizione dal latino, Roma, Carocci, 2000. 